Honam Mathematical J. 35 (2013), No. 2, pp. 319-327

http://dx.doi.org/10.5831/HMJ.2013.35.2.319

\title{
THE EQUIVALENCE CONDITIONS FOR SEMIALGEBRAICALLY PROPER MAPS
}

\author{
Dae Heui PARK
}

\begin{abstract}
In this paper we compare the notion of proper map in the category of topological spaces with that in the category of semialgebraic sets. To do this, we find some equivalence conditions for semialgebraically proper maps. In particular, we prove that a continuous semialgebraic map is semialgebraically proper if and only if it is proper. Moreover, we compare the semialgebraically proper map with the proper map in the sense of Delfs and Knebush [4].
\end{abstract}

\section{Introduction}

The purpose of this paper is to compare the notion of proper map in the category of topological spaces with that in the category of semialgebraic sets.

A semialgebraic set is a subset of $\mathbb{R}^{n}$ defined by finite number of polynomial equations and inequalities. More precisely, a subset $X$ of $\mathbb{R}^{n}$ is semialgebraic if and only if there exist polynomials $f_{i j}$ and $g_{i j}$ for $i=1, \ldots, p$ and $j=1, \ldots, q$ such that

$$
X=\bigcup_{i=1}^{p}\left\{x \in \mathbb{R}^{n} \mid f_{i j}(x)>0, g_{i j}(x)=0 \text { for all } j=1, \ldots, q\right\} .
$$

Throughout this paper we consider semialgebraic sets in $\mathbb{R}^{n}$ equipped with the subspace topology induced by the usual topology of $\mathbb{R}^{n}$. A semialgebraic space is an object obtained by pasting finitely many semialgebraic sets together along open semialgebraic subsets. Usually, a semialgebraic set in $\mathbb{R}^{n}$ is called an affine semialgebraic space over the real numbers. Since the topology of a semialgebraic space induced by those of finitely many semialgebraic sets, the topologies of semialgebraic

Received May 9, 2013. Accepted May 21, 2013.

2010 Mathematics Subject Classification. 14P10, 54C10.

Key words and phrases. semialgebraic set, semialgebraic map, proper map. 
spaces are of no interest. For this reason, in this paper we will only treat the semialgebraic sets in $\mathbb{R}^{n}$.

A map $f: X \rightarrow Y$ between semialgebraic sets $X \subset \mathbb{R}^{m}$ and $Y \subset \mathbb{R}^{n}$ is called semialgebraic if its graph is a semialgebraic set in $\mathbb{R}^{m+n}$.

In this paper we find the equivalence conditions for semialgebraically proper maps. Let $X$ and $Y$ be semialgebraic sets. A semialgebraic map $f: X \rightarrow Y$ is called semialgebraically closed if $f$ maps every closed semialgebraic subset of $X$ to a closed semialgebraic subset of $Y$. Similarly, $f$ is called semialgebraically proper if the preimage $f^{-1}(C)$ is compact for every compact semialgebraic subset $C$ of $Y$. Since $C$ should be semialgebraic in the definition, this notion is weaker than the condition that $f$ is proper. However, in this paper, we prove the following two theorems.

Theorem 1.1. Let $X$ and $Y$ be semialgebraic sets, and let $f: X \rightarrow Y$ be a semialgebraic map. Suppose $f$ is continuous. Then the following are equivalent:

(1) $f$ is semialgebraically proper;

(2) $f$ is semialgebraically closed and its fibers are compact.

Theorem 1.2. Let $X$ and $Y$ be semialgebraic sets, and let $f: X \rightarrow Y$ be a semialgebraic map. Suppose $f$ is continuous. Then the following are equivalent:

(1) $f$ is semialgebraically proper;

(2) $f$ is proper.

This paper is organized as follows. In Section 2 we develop the theory of semialgebraically proper maps. It also contains the proofs of Theorems 1.1 and 1.2. In Section 3 we compare the semialgebraically proper map with the "proper" map in the sense of Delfs and Knebush [4].

\section{Semialgebraically proper maps}

In this section we find the equivalence conditions for semialgebraically proper maps. It also contains the proofs of Theorems 1.1 and 1.2.

We first introduce well known facts on proper maps without proofs. For the details, we refer the reader to [2] and [5].

Let $X$ and $Y$ be a topological spaces, and $f: X \rightarrow Y$ a map which is not necessarily continuous. $f$ is called proper if the preimage of each compact subset of $Y$ is compact. $f$ is called closed if the image of each closed subset of $X$ is closed in $Y$. 
Proposition 2.1. Let $X$ and $Y$ be topological spaces. If $f: X \rightarrow Y$ is a closed map with compact fibers, then $f$ is proper.

A topological space is called compactly generated if it has the following property: if $A$ is any subset of $X$ whose intersection with each compact subset $K$ of $X$ is closed in $K$, then $A$ is closed in $X$.

It is well known fact that first countable spaces and locally compact spaces are compactly generated. Moreover, metric spaces, manifolds, and subsets of manifolds are compactly generated.

Proposition 2.2. Let $X$ be a topological space and $Y$ a compactly generated Hausdorff space. If $f: X \rightarrow Y$ is a continuous proper map, then $f$ is a closed map.

We now gather some properties concerning semialgebraic sets and maps without proof. For the details, we refer the reader to [1] and [4].

It is well known that finite union, finite intersection, and the cartesian product of semialgebraic sets are also semialgebraic. The composition of two semialgebraic maps is also semialgebraic. Moreover, the image and the preimage of a semialgebraic subset by a semialgebraic map are semialgebraic. In particular, let $X$ and $Y$ be semialgebraic sets, then the projection $p: X \times Y \rightarrow X$ defined by $p(x, y)=x$ is semialgebraic.

Proposition 2.3. Let $X$ be a semialgebraic set.

(1) If $A$ is a semialgebraic subset of $X$, then the closure $\bar{A}$, the complement $A^{c}$ and the interior $A^{\circ}$ in $X$ are semialgebraic.

(2) If $V$ is a neighborhood of a point $x$ in $X$, then there is a semialgebraic neighborhood $U$ of $X$ with $x \in \bar{U} \subset V$.

The following is an almost immediate consequence of the definitions.

Lemma 2.4. Let $X$ and $Y$ be semialgebraic sets, and let $f: X \rightarrow Y$ be a semialgebraically proper map.

(1) For a closed semialgebraic subset $A$ of $X$, the restriction $\left.f\right|_{A}: A \rightarrow f(A)$ is semialgebraically proper.

(2) For a semialgebraic subset $B$ of $Y$, the restriction $\left.f\right|_{f^{-1}(B)}: f^{-1}(B) \rightarrow B$ is semialgebraically proper.

(3) If $A$ is a semialgebraic subset of $X$ such that $A=f^{-1}(f(A))$, then the restriction $\left.f\right|_{A}: A \rightarrow f(A)$ is semialgebraically proper.

As a sequence plays an important role in the category of metric spaces, a curve germ plays in the semialgebraic category, see [1] or [3]. A curve germ in a semialgebraic set $X$ is represented by a "continuous" semialgebraic map $\alpha:(0, \epsilon] \rightarrow X$ for some $\epsilon>0$. Two curve germs are 
considered the same if, after possible reparameterization of the intervals, they agree on a common subinterval $(0, \delta]$ for some $\delta>0$. Thus, a curve germ is determined by the collection of images sets $\alpha((0, \epsilon]) \subset X$ for $\epsilon>0$. If a curve germ $\alpha$ extends to a continuous map $\alpha:[0, \epsilon] \rightarrow X$, we say the extension is the completion of $\alpha$, and $\alpha$ is completable. We write $\alpha \rightarrow x$ if $\alpha$ has a completion with $\alpha(0)=x$.

If $\alpha:(0, \epsilon] \rightarrow X$ has a completion with $\alpha \rightarrow x$, then given any neighborhood $U$ of $x$ in $X$, by restricting to a smaller interval whenever necessary, we may assume that $\alpha([0, \epsilon]) \subset U$.

We state the following elementary propositions because it will be used in this paper.

Proposition 2.5 ([3, p.73]). Let $X$ and $Y$ be semialgebraic sets.

(1) Every curve germ in a compact semialgebraic set has a completion.

(2) If $x$ belongs to the closure of a semialgebraic subset $A$ of $X$, then there is a curve germ $\alpha$ in $A$ with $\alpha \rightarrow x$.

(3) Every curve germ in $X \cup Y$ is a curve germ in either $X$ or $Y$.

From now on, $X$ and $Y$ denote semialgebraic sets.

Proposition 2.6 ([3, p.73]). Let $f: X \rightarrow Y$ be a semialgebraic map.

(1) If $f: X \rightarrow Y$ is surjective, then every curve germ $\alpha$ in $Y$ lifts to a curve germ $\tilde{\alpha}$ in $X$, that is $f \circ \tilde{\alpha}=\alpha$.

(2) $f$ is continuous if and only if for any completable curve germ $\alpha$ in $X$ with $\alpha \rightarrow x$, the curve germ $f \circ \alpha$ is also completable in $Y$ with $f \circ \alpha \rightarrow f(x)$.

(3) If $f$ is semialgebraically proper, then for any curve germ $\tilde{\alpha}$ in $X$ such that $f \circ \tilde{\alpha}$ is completable in $Y$ is completable in $X$. Moreover, the converse holds if $f$ is continuous.

As in the category of topological spaces, we have the following proposition.

Proposition 2.7. Let $X$ be a semialgebraic set. Then the following are equivalent:

(1) $X$ is compact;

(2) for any semialgebraic set $Y$, the projection $p: X \times Y \rightarrow Y$ defined by $p(x, y)=y$ is semialgebraically closed;

(3) the projection $p: X \times I \rightarrow I$ defined by $p(x, y)=y$ is semialgebraically closed, where $I$ denotes the unit interval $[0,1]$ equipped with the subspace topology induced by the usual topology of $\mathbb{R}$. 
Proof. It suffices to show that (3) implies (1). Suppose $X$ is not compact. We can find a continuous semialgebraic map $\alpha:[0,1) \rightarrow X$ such that the image $\alpha([0,1))$ is semialgebraic and closed in $X$. Then the set $A=\{(\alpha(t), t) \mid 0 \leqq t<1\}$ is semialgebraic and closed in $X \times I$. But the image $p(A)=[0,1)$ is not closed in $I$. It follows that $p$ is not semialgebraically closed. This completes the proof.

Propositions 2.1 and 2.2 are still valid in the semialgebraic category as in the following two propositions. We remark that all semialgebraic sets are metric spaces and therefore compactly generated Hausdorff.

Proposition 2.8. Let $f: X \rightarrow Y$ be a semialgebraic map. If $f$ is a semialgebraically closed map with compact fibers, then $f$ is semialgebraically proper.

Proof. Let $K$ be a compact semialgebraic subset of $Y$. Let $\left\{U_{\alpha} \mid \alpha \in\right.$ $\Lambda$ \} be a cover of $f^{-1}(K)$ by open subsets of $X$. For $x \in U_{\alpha}$ we can take an open semialgebraic set $W_{\alpha, x}$ such that $x \in W_{\alpha, x} \subset U_{\alpha}$. Then the collection $\mathcal{B}=\cup_{\alpha \in \Lambda}\left\{W_{\alpha, x} \mid x \in U_{\alpha}\right\}$ is a refinement of $\left\{U_{\alpha} \mid \alpha \in \Lambda\right\}$. It suffices to show that $f^{-1}(K)$ is covered by finitely many of the sets in $\mathcal{B}$.

For $y \in K$, the fiber $f^{-1}(y)$ is compact, so it is covered by finitely many of the sets in $\mathcal{B}$. In other words, for $y \in K$, there is a finite subset $\mathcal{B}_{y}$ of $\mathcal{B}$ such that $f^{-1}(y) \subset \cup_{W \in \mathcal{B}_{y}} W$. Then the set $C_{y}=X \backslash \cup_{W \in \mathcal{B}_{y}} W$ is closed and semialgebraic in $X$ and disjoint from $f^{-1}(y)$. Since $f$ is semialgebraically closed, the set $V_{y}=Y \backslash f\left(C_{y}\right)$ is open and semialgebraic in $Y$ and contains $y$. It is easy to check that $f\left(V_{y}\right) \subset \cup_{W \in \mathcal{B}_{y}} W$ and that $\left\{V_{y} \mid y \in K\right\}$ is a cover of $K$. Because $K$ is compact, it is covered by finitely many of the sets $V_{y}$. Thus $f^{-1}(K)$ is covered by finitely many sets of the form $f^{-1}\left(V_{y}\right)$, each of which is covered by finitely many of the sets in $\mathcal{B}$, so it follows that $f^{-1}(K)$ is compact.

Proposition 2.9. Let $f: X \rightarrow Y$ be a semialgebraic map. Suppose $f$ is continuous. If $f$ is semialgebraically proper, then $f$ is semialgebraically closed.

Proof. Let $f$ be a semialgebraically proper map which is continuous. Suppose $C$ is closed and semialgebraic in $X$. Clearly, the image $f(C)$ is semialgebraic in $Y$. It suffices to show that $f(C)$ is closed in $Y$. Let $\overline{f(C)}$ denote the closure of $f(C)$ in $Y$. Given $y \in \overline{f(C)}$, it follows from Proposition 2.5(2) that there exists a curve germ $\alpha$ in $f(C)$ with $\alpha \rightarrow y$. Since the restriction $\left.f\right|_{C}: C \rightarrow f(C)$ is surjective, by Proposition 2.6(1), there is a curve germ $\tilde{\alpha}$ in $C$ such that $f \circ \tilde{\alpha}=\alpha$. By Proposition 2.6(3), 
$\tilde{\alpha}$ is completable in $X$. Let $\tilde{\alpha} \rightarrow x$, then $x \in C$ because $C$ is closed in $X$. By Proposition 2.6(2), $\alpha=f \circ \tilde{\alpha} \rightarrow f(x)$, and hence $y=f(x) \in f(C)$. Therefore $f(C)$ is closed in $Y$.

From Propositions 2.8 and 2.9 we have Theorem 1.1.

Let $f: X \rightarrow Y$ and $g: Z \rightarrow Y$ be semialgebraic maps. Then the pullback $X \times_{Y} Z=\{(x, z) \in X \times Z \mid f(x)=g(x)\}$ is semialgebraic and the pullback diagram

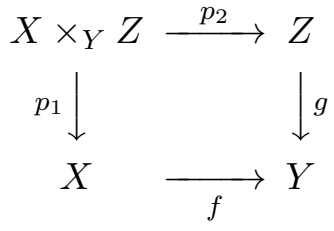

commutes, where $p_{1}$ and $p_{2}$ are the canonical projections, that is $p_{1}(x, z)=x, p_{2}(x, z)=z$. We remark that $p_{1}$ and $p_{2}$ are continuous and semialgebraic.

Proposition 2.10. Let $f: X \rightarrow Y$ be a semialgebraic map. Then the following are equivalent:

(1) $f$ is semialgebraically proper;

(2) for any semialgebraic set $Z$ and any continuous semialgebraic map $g: Z \rightarrow Y$, the projection $p_{2}: X \times_{Y} Z \rightarrow Z$ is semialgebraically proper.

Proof. Let $f$ be a semialgebraically proper map and $g: Z \rightarrow Y$ a continuous semialgebraic map. If $K$ is a compact semialgebraic subset of $Z$, then the preimage $p_{2}^{-1}(K)$ is a closed subset of a compact set $f^{-1}(g(K)) \times K$, so it is compact. It follows that $p_{2}$ is semialgebraically proper.

Conversely, we consider the identity map $g: Y \rightarrow Y, g(y)=y$. Then the pullback $X \times_{Y} Y$ is the graph of $f$. Suppose $K$ is a compact semialgebraic subset of $Y$. Because $p_{2}$ is semialgebraically proper, the set $p_{1}\left(p_{2}^{-1}(K)\right)$ is compact and semialgebraic, this set is equal to $f^{-1}(K)$. It follows that $f$ is semialgebraically proper.

Lemma 2.11. Let $f: X \rightarrow Y$ be a semialgebraic map. If $f$ is semialgebraically closed with compact fibers, then $f$ is closed.

Proof. Let $C$ be a closed subset of $X$. Suppose $f(C)$ is not closed in $Y$. Then there exist a point $y$ in $\overline{f(C)}$ which is not contained in $f(C)$. Since $f^{-1}(y)$ is disjoint from the closed set $C$, for every point $x \in f^{-1}(y)$ has a 
semialgebraic neighborhood $U_{x}$ which does not meet $C$. Since $f^{-1}(y)$ is compact, there exist finitely many points $x_{1}, \ldots, x_{n} \in f^{-1}(y)$ such that

$$
f^{-1}(y) \subset U_{x_{1}} \cup \cdots \cup U_{x_{n}} .
$$

Then the set $B=X \backslash\left(U_{x_{1}} \cup \cdots \cup U_{x_{n}}\right)$ is closed and semialgebraic in $X$ and contains $C$. Since $f$ is semialgebraically closed, the image $f(B)$ is closed in $Y$. Thus $\overline{f(C)} \subset f(B)$. This contradiction since $y \notin f(B)$. Therefore $f$ is closed.

Let us now prove Theorem 1.2.

Proof of Theorem 1.2. Let $f: X \rightarrow Y$ be a continuous semialgebraic map. Clearly, we see that if $f$ is proper, then it is semialgebraically proper. The converse follows from Theorem 1.1, Lemma 2.11 and Proposition 2.1.

Proposition 2.12. Let $f: X \rightarrow Y$ be a semialgebraic map. Suppose $f$ is continuous. Then the following are equivalent:

(1) $f$ is semialgebraically proper;

(2) for any semialgebraic set $Z$ and any continuous semialgebraic map $g: Z \rightarrow Y$, the projection $p_{2}: X \times_{Y} Z \rightarrow Z$ is semialgebraically closed.

(3) for any semialgebraic set $Z$, the map $f \times \operatorname{id}_{Z}: X \times Z \rightarrow Y \times Z$ defined by $f \times \operatorname{id}_{Z}(x, z)=(f(x), z)$ is semialgebraically closed.

Proof. (1) $\Rightarrow(2)$ This follows from Propositions 2.9 and 2.10 .

$(2) \Rightarrow(3)$ Assume $f$ satisfies (2). Given any semialgebraic set $Z$, consider the continuous semialgebraic map $g: Y \times Z \rightarrow Y$ defined by $g(y, z)=y$, then we have $X \times_{Y}(Y \times Z)=\{(x, f(x), z) \mid x \in X, z \in Z\}$. Define $h: X \times Z \rightarrow X \times \times_{Y}(Y \times Z)$ by $h(x, z)=(x, f(x), z)$. Then $h$ is a semialgebraic homeomorphism, and hence semialgebraically closed. By assumption, the projection $p_{2}: X \times_{Y}(Y \times Z) \rightarrow Y \times Z$ is also semialgebraically closed. It is easy to check that $f \times \operatorname{id}_{Z}=p_{2} \circ h$, so the map $f \times \operatorname{id}_{Z}$ is semialgebraically closed.

$(3) \Rightarrow(1)$ Assume $f$ satisfies (3). Let $K$ be a compact semialgebraic subset of $Y$, and let $Z$ be any semialgebraic set. By assumption, the map $f \times \operatorname{id}_{Z}: X \times Z \rightarrow Y \times Z$ is semialgebraically closed, and thus the restriction $f \mid \times \operatorname{id}_{Z}: f^{-1}(K) \times Z \rightarrow K \times Z$ of $f \times \mathrm{id}_{Z}$ is semialgebraically closed. Because $K$ is a compact semialgebraic set, the projection $p: K \times Z \rightarrow Z, p(x, z)=z$ is semialgebraically closed by Proposition 2.7. Hence the composition $p \circ\left(f \mid \times \operatorname{id}_{Z}\right): f^{-1}(K) \times Z \rightarrow Z$, $(x, z) \mapsto z$ is also semialgebraically closed, so $f^{-1}(K)$ is compact by Proposition 2.7. Therefore $f$ is semialgebraically proper. 


\section{Some remarks}

In this section we compare the semialgebraically proper map with the "proper" map in the both senses of Delfs and Knebush [4] and Scheiderer [7].

Let $X$ and $Y$ be semialgebraic sets, and let $f: X \rightarrow Y$ be continuous and semialgebraic. Delfs and Knebush [4] call $f$ "proper" if for any semialgebraic set $Z$ and for any continuous semialgebraic map $g: Z \rightarrow Y$, the projection $p_{2}: X \times_{Y} Z \rightarrow Z, p_{2}(x, z)=z$ is semialgebraically closed. We need to distinguish this notion from former(proper, semialgebraically proper). To to this, in this case we call $f$ DK-proper.

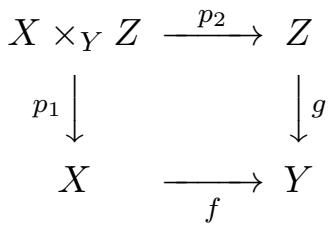

A semialgebraic set $X$ is called complete (in the sense of $[4,7]$ ) if the constant map from $X$ to the one point set is DK-proper. This means that for any semialgebraic set $Z$ the projection $p_{2}: X \times Z \rightarrow Z, p_{2}(x, z)=z$ is semialgebraically closed.

A semialgebraic set $X$ is called locally complete (in the sense of $[4,7]$ ) if every point of $X$ has a semialgebraic neighborhood which is complete.

Proposition 3.1. (1) A semialgebraic set is compact if and only if it is complete.

(2) A semialgebraic set is locally compact if and only if it is locally complete.

(3) A continuous semialgebraic map is semialgebraically proper if and only if it is DK-proper.

Proof. (1) This follows from Proposition 2.7.

(2) Let $X$ be a semialgebraic set in $\mathbb{R}^{n}$. Suppose $X$ is locally complete. Then every $x \in X$ has a semialgebraic neighborhood $U$ which is complete. By (1), $U$ is compact. Therefore $X$ is locally compact.

Conversely, suppose $X$ is locally compact. Then every $x \in X$ has a compact neighborhood $U$ in $X$. Clearly, there is a positive real number $r$ such that $B(x, r) \cap X \subset U$, where $B(x, r)$ is the open ball of radius $r$ centered at $x$ in $\mathbb{R}^{n}$. Let $V$ be the closure of $B(x, r) \cap X$ in $X$. Then $V$ is a semialgebraic neighborhood of $x$ in $X$ which is compact. By (1), $V$ is complete. Therefore $X$ is locally complete.

(3) This follows from Proposition 2.12. 
Remark 3.2. Recall that a semialgebraic space is an object obtained by pasting finitely many semialgebraic sets together along open semialgebraic subsets. R. Robson proved in [6] that every regular semialgebraic space admits a semialgebraic embedding into $\mathbb{R}^{n}$. More precisely, for a regular semialgebraic space $X$, there exist a semialgebraic set $Y$ in $\mathbb{R}^{n}$ and a semialgebraic homeomorphism $f: X \rightarrow Y$. From this we see that the results in this paper are still valid for regular semialgebraic spaces.

\section{References}

[1] J. Bochnak, M. Coste and M.-F. Roy, Real Agebraic Geometry, Erg. der Math. und ihrer Grenzg., vol. 36, Springer-Verlag, Berlin Heidelberg, 1998.

[2] N. Bourbaki, General Toplogy, Assison-Wesley, 1966.

[3] G. W. Brumfiel, Quotient space for semialgebraic equivalence relation, Math. Z. 195 (1987), 69-78.

[4] H. Delfs and M. Knebusch, Semialgebraic topology over a real closed field II: Basic theory of semialgebraic spaces, Math. Z. 178 (1981), 175-213.

[5] J. Dugundji, Toplogy, Allyn and Bacon, Boston, 1966.

[6] R. Robson, Embedding semi-algebraic spaces, Math. Z. 183 (1983), 365-370.

[7] C. Scheiderer, Quotients of semi-algebraic spaces, Math. Z. 201 (1989), 249-271.

Dae Heui Park

Department of Mathematics,

College of Natural Sciences, Chonnam National University,

Gwangju 500-757, Korea.

E-mail: dhpark3331@jnu.ac.kr 